\title{
Effect of Spinal Exercises and Epidural Injections in Chronic Low Back Pain
}

\author{
Sargun Singh Walia, Jaswinder Pal Singh, Harshita Yadav and Anuradha Lehri
}

\begin{abstract}
Aim: Effect of Spinal Exercises and Epidural Injections in Chronic Low Back Pain Method: A total of 50 patients were given epidural injection with 18 males and 32 females. The patient who was included in the study should be diagnosed be chronic low back pain with or without radicular pain, age group 19-70 years, should have pain more than three months. The outcome measures were pain scale and Ronald Morris Disability Questionnaire for pain and disability respectively. Results: The result suggested that there is significant decrease in the pain, after the injection at all points, when follow up were taken after one month, after three months and after six months with $\mathrm{p}$ value $<0.0001$. Also, there are significant results for RMDQ showing decrease in disability after injection at all the points, when follow up were taken after one month, after three months, after six months with $\mathrm{p}$ value $=0.0001$. Conclusion: The study concluded that epidural steroid injections along with spinal exercises were effective in reducing pain and disability in chronic low back pain patient.
\end{abstract}

\author{
Sargun Singh Walia \\ Emergency Consultant \\ Columbia Asia Hospital, Patiala (Punjab) India \\ E mail- drsargunsingh@gmail.com \\ Jaswinder Pal Singh \\ Chief Orthopaedic Surgeon \\ Aggarasain Hospital, Patiala(Punjab) India \\ Harshita Yadav \\ Ph.D. Researcher \\ Department of Sports science \\ Punjabi University, Patiala(Punjab) India \\ Anuradha Lehri \\ Assistant Professor \\ Department of Sports Science \\ Punjabi University Patiala (Punjab) India
}

\author{
Key Words: Back, Epidural, Exercise, \\ Pain, Spinal
}

DOI: 10.18376/jesp/2017/v13/i1/111277

\section{Introduction}

Low back pain (LBP) is one of the most common public health problems worldwide with a leading cause of activity limitation. In India, the incidence of LBP has been reported to be $23.09 \%$ and has a lifetime prevalence ranges between $6.2 \%$ (general population) to $92 \%$ (constructional workers) (Bindra et al, 2015; Thakur \& Mahapatra, 2015). It is more prevalent among women as compared to men and incidence increases with age. It is estimated that about $60-80 \%$ of population have LBP at some point in their life and it affects all ages (EL Desoky). The etiological factors usually contributes to LBP are altered lifestyle, increased tensions in life, lack of physical exercises, prolonged sedentary lifestyle, occupation which requires prolong sitting hours as in computer professionals, bad postures, overeating, heavy weight lifting (Wheeler \& Murrey, 2005). LBP has classified on the basis of: pathoanatomy, presence or absence of specific signs or symptoms, duration of symptoms (acute, sub acute or chronic), work status, diagnostic testing, patient history, 


\section{Journal of Exercise Science \& Physiotherapy, Vol. 13, No. 1, 2017 \\ ISSN: 0973-2020 (Print) $\quad \mathrm{I}_{2}$ OR Impact Factor $=5.23 \quad$ UGC Approved [no.20489] ISSN: 2454-6089 \\ (Online)}

or combination of these. LBP is defined as chronic after three months as the normal tissue heal within 6-12 weeks, unless patho-anatomic instability persists. Chronic LBP might originate from degenerative conditions of spine (Waddell 1978). There are numerous conditions which can results in chronic low back pain with or without radiating symptoms like prolapsed intervertebral disc, spondylolisthesis, lumbar spondylosis, spinal stenotic syndrome, osteoarthropathy. Most of conditions are dealt with either of the therapeutic approaches such as bed rest, drug therapy, acupuncture, spinal cord stimulation, cryotherapy, radiotherapy, thermocoagulation, psychotherapy, spinal exercises and surgery (Kang et al., 2011). The epidural injection of steroid is gaining much attention in the modern era. It is also advantageous over the systemic therapy, more over of its higher concentration of drug delivered to diseased area and also with its low rate of systemic adverse effects. The use of first epidural injection was reported in 1953, for the treatment of axial and radicular back pain (Sung 2006). Epidural injection is given by accessing the lumbar epidural space by multiple routes including caudal, transforaminal and interlaminer (Parr 2012). Both interlaminer and caudal epidural injections require relatively large volume of injectants for delivering steroids to target site. While, the transforaminal approach is considered the targetspecific modality requiring the smallest volume to reach the primary site of pathology (Sung 2006). The image intensified guided epidural steroid injections are more popular as they overcome the problem of lacking target specificity. There are very few clinical trials that are evident for determining the effect of epidural steroid injection along with spinal exercises.

\section{Materials and Methods}

A total of 50 patients diagnosed with chronic low back pain were recruited in the study. All the patients were taken from Aggarasain hospital, Patiala. The diagnosis of the patient was confirmed by radiological investigations. The patient who was included in the study should be diagnosed be chronic low back pain with or without radicular pain (with clinical examination, x-ray/MRI), age group 19-70 years, should have pain more than three months; both males and females, pain grading more than 3 on pain scale, positive straight leg raise test, written consent was taken from the patient. A total of 18 males and 32 females are included. All those who have history of allergic reaction to local anesthetics and steroids, known contra-indications of epidural steroids, history of epidural steroid injection within past six months. All patient, were given a set of spinal exercises along with the epidural steroid injection. The outcome measure used in the study were pain and disability, measured before giving the injection (baseline) and after giving injection i.e first after one month, three months, after six months. The severity of pain and disability was assessed by grading on Pain scale and Ronald Morris Disability Questionnaire (RMDQ) respectively.

Spinal Exercises

A set of spinal exercises include single and double knee to chest, pelvic rolling, and pelvic bridging, prone on elbows, prone on hands. All the exercises were sequentially introduced in the protocol with 20 repetitions of each exercise. Total 30 minutes protocol was set.

\section{Epidural Injection procedure}

The patient was asked to lie down in prone position, injection through lumbosacral route. The injection of methylprednisolone $(80 \mathrm{mg})$, xylocaine $(2 \mathrm{ml})$, normal saline $(10 \mathrm{cc})$. Patient's pulse rate and blood pressure was monitored every 15 minutes for 1 hour. Also, the patients were discharged on the same day once they had voided the urine. The epidural steroid injection was repeated if necessary depending upon patient's pain relief.

The patients were again clinically examined for the neurological deficits. The follow ups were taken at an interval of one month, three month and six month. 


\section{Results}

The statistical analysis for the data was done using the graph pad. The statistical test used in the present study was paired t-test. Table 1 and table 2 showing the pain scale and RMDQ scores respectively. Mean, standard deviation, $\mathrm{t}$-value and $\mathrm{p}$-value was analyzed for the duration before injection and after one month, before injection and after three months, before injection and after six months.

The result suggested that there is significant decrease in the pain, after the injection at all points, when follow up were taken after one month, after three months and after six months with $p$ value $<0.0001$. Also, there are significant results for RMDQ showing decrease in disability after injection at all the points, when follow up were taken after one month, after three months, after six months with $\mathrm{p}$ value $=0.0001$.

Table1. Pain Scale before injection, after one-month, after 3 months, and after 6 months

\begin{tabular}{llcccc}
\hline Duration & Pain Scale & Mean \pm SD & Mean Difference \pm SD & t-value & p-value \\
\hline \multirow{2}{*}{ Baseline- 1 month } & Before Injection & $5.98 \pm 1.317$ & & & \\
& After one month & $1 \pm 1.565$ & $4.980 \pm 0.8919$ & 39.481 & $<0.0001$ \\
\hline & Before Injection & $5.98 \pm 1.317$ & & & \\
Baseline- 3 month & After 3 month & $1.36 \pm 1.562$ & $4.4620 \pm 0.8545$ & 38.230 & $<0.0001$ \\
& Before Injection & $5.98 \pm 1.317$ & & & \\
\hline & & & & & \\
Baseline- 6 month & After 6 month & $1.52 \pm 1.607$ & & & \\
\hline
\end{tabular}

Table 2. RMDQ score before injection, after one month, after 3 month and 6 month

\begin{tabular}{|c|c|c|c|c|c|}
\hline Duration & RMDQ & Mean \pm SD & Mean Difference \pm SD & t-value & p-value \\
\hline \multirow{3}{*}{ Baseline- 1 month } & Before Injection & $21.22 \pm 2.509$ & \multirow{3}{*}{$16.480 \pm 3.382$} & \multirow{3}{*}{34.456} & \multirow{3}{*}{0.0001} \\
\hline & & & & & \\
\hline & After one month & $4.74 \pm 3.917$ & & & \\
\hline \multirow{3}{*}{ Baseline- 3 month } & Before Injection & $21.22 \pm 2.509$ & \multirow{3}{*}{$14.920 \pm 5.030$} & \multirow{3}{*}{20.975} & \multirow{3}{*}{0.0001} \\
\hline & & & & & \\
\hline & After 3 month & $6.3 \pm 5.658$ & & & \\
\hline \multirow{3}{*}{ Baseline- 6 month } & Before Injection & $21.22 \pm 2.509$ & \multirow{3}{*}{$14.460 \pm 4.945$} & \multirow{3}{*}{20.675} & \multirow{3}{*}{0.0001} \\
\hline & & & & & \\
\hline & After 6 month & $6.76 \pm 5.847$ & & & \\
\hline
\end{tabular}




\section{Journal of Exercise Science \& Physiotherapy, Vol. 13, No. 1, 2017 \\ ISSN: 0973-2020 (Print) I2OR Impact Factor $=5.23$ UGC Approved [no.20489] ISSN: 2454-6089 \\ (Online)}

\section{Discussion}

The present study evaluated the effect of epidural injection with spinal exercises for decreasing pain and disability in cLBA patients. The result of the present study suggested that mean for pain and disability was high before injection with the spinal exercises $(5.98 \pm 1.317)$. The patients' pain score has significantly decreased at all the points, that is when scoring is done after one-month, after three months and six month with mean value of $1 \pm 1.565,1.36 \pm 1.562$ and $1.52 \pm 1.607$ (p< 0.0001 ).respectively. The greatest difference was found after one month which was $4.980 \pm 0.8919$ $(\mathrm{p}<0.0001)$. The disability of all patients was high before injection with spinal exercises with a mean 21.22 \pm 2.509 . When the patients were given injection, the score of disability was found to be decreased markedly at all the points that are after one month, after three months, after six months with a mean score of $4.74 \pm 3.917,6.3 \pm 5.658$ and $6.76 \pm 5.847$ respectively $(\mathrm{p}<0.0001)$. The maximum difference found in disability was after one month with a score of $16.480 \pm 3.382(\mathrm{p}<$ 0.0001). The result of the present study was somewhat similar with a study conducted by Manchikanti et al. in which they concluded that both caudal epidural injection of local anesthetic with or without steroids are effective in patients with chronic axial low back pain ( of discogenic origin without facet joint pain, disc herniation and or radiculitis). Song et al. conducted a clinical trial to evaluate the effect and safety of steroid injection in lumbar spinal stenosis: with or without local anesthetics. The results concluded that Visual analogue scale and functional rate index were reduced significantly in both local anesthetic group and saline group. Thus, the spinal injection procedures using steroids mixed either with local anesthetics or normal saline have an effect in reducing pain and improving functional activities. However, there was no significant difference between the two groups in relation to side effects and the long-term effects of pain and function. Rosenberg et al evaluated the effectiveness of transforaminal epidural steroid injections in low back pain and follow up of patient was taken over a period of one year. Ninety-two patients with radiculopathic back pain due to spinal stenosis, herniated discs, spondylolisthesis, and degenerated discs, underwent transforaminal epidural steroid injections under fluoroscopy. The results suggested that pain scores for all patients improved significantly at all three time points (2, 6 and 12 months) compared to the initial mean pain score. The study concluded that transforaminal epidural steroid injections can offer significant pain reduction up to one year after initiation of treatment in patients with discogenic pain and possibly in patients with spinal stenosis. Epidural steroid injections have been widely used to treat radiculopathic pain, with varying degrees of effectiveness. The present study found a marked reduction in patient's pain and disability due to chronic back pain after implication of epidural injection along with spinal exercises.

\section{Conclusion}

The study concluded that pain and disability has been decreased with spinal exercises along with the epidural steroid injection in chronic low back pain patients.

\section{References}

Bindra, S., Sinha, A.G.K., Benjamin, A.I. (2015). Epidemiology of Low Back Pain in Indian Population: A Review. International Journal of Basic and Applied Medical Sciences. 5(1):166-79.

ELDesoky, M.T.M., \& Abutaleb, E.E.M. (2016). Efficacy of Neural Mobilization on Low Back Pain with S1 Radiculopathy. Int J Physiother. 3(3): 362-70.

Kang, S.S., Hwang, B.M., Son, H.J., Cheong, Y., Lee, S.J., Lee, S.H., Chung, T.Y. 2011. The Dosages of Corticosteroid in Transforaminal Epidural Steroid Injections for Lumbar Radicular Pain Due to a Herniated Disc. Pain Physician. 14:361-370. 
Manchikanti, L., Cash, K.A., McManus C.D., Pampati, V. 2012. Fluoroscopic caudal epidural injections in managing chronic axial low back pain without disc herniation, radiculitis, or facet joint pain. J. pain Research. 5:381-390.

Parr, A.T., Manchikanti, L., Hameed, H., Conn, A., Manchikanti, K.N., Benyamin, R.N., Diwan, S., Singh, V., \& Abdi, S. 2012. Caudal Epidural Injections in the Management of Chronic Low Back Pain: A Systematic Appraisal of the Literature. Pain Physician. 15:E159-E198.

Rosenberg, S.K., Grabinsky, A., Kooser, C., \& Boswell, M.V. 2002. Effectiveness of Transforaminal Epidural Steroid Injections in Low Back Pain: A One-Year Experience Pain Physician, 5(3):266-270

Song, S.H., Ryu, G.H., Park, J.W., Lee. J.H., Nam, K.Y., Kim, H., Kim, S.Y., Kwon, B.S. 2016. The Effect and Safety of Steroid Injection in Lumbar Spinal Stenosis: With or Without Local Anesthetics Ann Rehabil Med. 40(1):14-20

Sung, M.S., 2006. Epidural Steroid Injection for Lumbosacral Radiculopathy. Korean J Radiol. 7(2). 77-79

Thakur, A., \& Mahapatra R.K. (2015). Effect of Mulligan Spinal Mobilization with Leg Movement and Shacklock Neural Tissue Mobilization in Lumbar Radiculopathy: A Randomised Controlled Trial. Journal of Medical Thesis. 3(2):27-30.

Conflict of Interest: None declared 